\title{
Projeto Aprenda a Programar Jogando: Divulgando a Programação de Computadores para Crianças e Jovens
}

\author{
Julia Godinho, Kathleen Torres, Gabriel Batista, \\ Eduardo Andrade, Janaína Gomide \\ ${ }^{1}$ Universidade Federal do Rio de Janeiro (UFRJ) - Campus Macaé \\ Macaé - RJ - Brazil \\ \{juliagodinho08,kathleent48,edu.gabriel23,gab.batista.10\}@gmail.com, \\ janainagomide@macae.ufrj.br
}

\begin{abstract}
This article introduces the research project Learn How to Program Playing and the experience resulting from the activities developed in it. The project aims to introduce the universe of computer programming to children and young people and encourage them to become technology creators. This article presents the tools selected for the teaching of programming, the structure of activities carried out both in the workshops and the mini courses and the plan of activities carried out in the mini courses. This project has already benefited 298 students and approximately 90\% rated the programming experience as excellent or great. In 2016, this project was recognized by the Brazilian Society of Computation (SBC).
\end{abstract}

Resumo. Este artigo apresenta o projeto Aprenda a Programar Jogando e a experiência resultante das atividades nele desenvolvidas. O projeto visa introduzir o universo da programação computacional a crianças e jovens e incentivá-los a serem criadores de tecnologia. Nesse artigo são apresentadas as ferramentas selecionadas para o ensino da programação, a estrutura de atividades realizadas tanto nas oficinas quanto nos minicursos e o plano de atividades realizados nos minicursos. Esse projeto já beneficiou 298 estudantes e aproximadamente $90 \%$ qualificaram a experiência com a programação como excelente ou ótima. Em 2016, o projeto recebeu Chancela da Sociedade Brasileira de Computação $(S B C)$.

\section{Introdução}

É cada vez mais comum o uso de tecnologias computacionais no dia a dia dos brasileiros, nas mais diversas atividades e finalidades, tais como no acesso a informação, na comunicação, no entretenimento ou em ferramentas de trabalho. Entretanto, a maioria das pessoas são ainda apenas usuários de softwares e aplicativos através de seus computadores e celulares, possuem habilidades para utilizá-los, mas não compreendem como são desenvolvidos e como funcionam.

No Brasil, o ensino da programação ainda é bastante restrito às universidades. A partir da observação desse cenário, que o projeto Aprenda a Programar Jogando foi elaborado, com objetivo de difundir o estudo da programação. O projeto visa levar aos alunos 
do ensino fundamental e médio de Macaé e região a oportunidade de ter o primeiro contato com a programação computacional, promovendo o entendimento básico do processo da criação de programas e aplicativos e o desenvolvimento do raciocínio lógico.

O conhecimento adquirido com a programação vai além de criar jogos, websites e sistemas. Quem entra em contato com esse universo desenvolve habilidade para resolução de problemas, criatividade e trabalho em equipe. Outros benefícios ligados ao domínio da programação é ajudar a organizar ideias, pensamentos e objetivos de vida [Wing 2006]. Nesse contexto, o ensino de conceitos de programação ainda na educação básica pode ser um grande facilitador para o processo de aprendizagem. Em particular, programação estimula o "pensamento computacional", ajudando na criação de soluções de problemas e desenvolvimento de estratégias, que possui utilidade além da área da ciência da computação [Resnick 2009].

As atividades oferecidas pelo projeto são oficinas e minicursos que utilizam ferramentas gratuitas disponíveis e previamente selecionadas pela equipe do projeto. A equipe do projeto é composta de alunos do curso de Engenharia Mecânica, Civil e Produção da UFRJ-Macaé e coordenados pela professora da área de Ciência da Computação. Em 2016, o projeto recebeu Chancela da Sociedade Brasileira de Computação (SBC) ${ }^{1}$ e todas as informações das atividades e tutoriais realizados estão disponíveis no site do projeto ${ }^{2}$.

O objetivo desse artigo é apresentar a estrutura de ensino elaborada no projeto e relatar a experiência obtida ao longo das atividades realizadas, de forma que possam ser replicadas e realizadas em outras instituições e escolas. As principais contribuições desse artigo contemplam a apresentação das ferramentas selecionadas para o ensino da programação para crianças e jovens, o desenvolvimento de uma estrutura de atividades a serem realizadas tanto nas oficinas quanto nas aulas dos minicursos e o desenvolvimento do plano de atividades a ser realizado nos minicursos para crianças e jovens.

O projeto já atingiu 298 crianças e jovens dos quais mais de $80 \%$ não sabiam como um programa de computador era feito e $100 \%$ não conheciam o que era ciência da computação ou linguagem de programação. Praticamente todos os alunos classificaram a experiência com a programação como sendo excelente ou ótima e indicariam as atividades para alguém.

\section{Trabalhos Relacionados}

Várias iniciativas têm sido realizadas no Brasil com o objetivo de difundir o aprendizado da computação, com o foco em diversos públicos, principalmente jovens.

O Programa Meninas Digitais, da Sociedade Brasileira da Computação (SBC), tem como objetivo divulgar a área de Computação para despertar o interesse de estudantes do ensino médio/tecnológico para que conheçam melhor a área e motivá-las a seguir carreira em Computação. O programa atua de diferentes formas: minicursos e oficinas; palestras com estudantes e profissionais que já atuam na área, dentre outros [SBC 2015]. Outro projeto com foco no público feminino é apresentado em [Ramos 2015] em que é apresentado um relato de experiência com alunas do ensino médio de uma escola estadual

\footnotetext{
${ }^{1} \mathrm{~A}$ chancela foi concedida para projetos que levem a computação até crianças, adolescentes e públicos que não tem contato com a área de computação aproximando a computação da sociedade brasileira.

${ }^{2}$ Link para o site do projeto: https://sites.google.com/site/projetoaprendaprogramar/
} 
e é utilizada a plataforma MIT App Inventor em suas atividades.

No artigo [Oliveira 2014] é relatada experiência de um grupo de extensão no ensino de conceitos básicos da ciência da computação feita com 11 alunos do ensino fundamental usando Scratch. Já em [França 2014] diversas ações de divulgação foram realizadas como participação em feiras de profissões, matérias para rádios e jornais locais, e visitas em escolas publicas e privadas. As atividades desse trabalho contemplam o ensino de número binários, uso do Scratch e ensino de robótica.

Uma experiência interessante relatada em [Silva 2016], propôs uma abordagem intitulada Saberes D'Avó, em que os alunos são colocados em contato com avós, em um minicurso, para discutir sobre saberes regionais e, por meio de um treinamento, criam aplicativos do tipo quiz na plataforma MIT App Inventor. A experiência foi feita com 16 alunos do ensino médio e o caráter lúdico da ferramenta proporcionou um ambiente confortável para tratar de assuntos complexos.

Em uma análise diferente do uso de jogos educacionais [Jakos 2016] afirma que o uso de jogos apropriados pode gerar um melhora no raciocínio lógico, na capacidade de aprendizado e no conhecimento de estudantes, que pode ser significativa o suficiente para permitir que os professores usem outras ferramentas de ensino, com complexidade superior aos métodos tradicionais. O estudo foi realizado com a implementação de jogos educacionais para o ensino de conhecimentos da computação feito com 107 alunos do sexta série.

O Projeto Aprenda a Programar Jogando se diferencia dos demais quanto a quantidade e tipo de ferramentas utilizadas e pelo plano estruturado de aulas para a utilização dessa ferramentas, que permite o beneficiamento de um grande público e de forma contínua. Também se diferencia quanto ao público, pois a metodologia proposta foi adequadamente elaborada para o ensino simultâneo de crianças e jovens, enquanto os trabalhos descritos anteriormente são para crianças ou jovens, independentemente. Por fim, o Projeto se diferencia por sua abrangência e capacidade de atingir um grande público.

\section{Público Alvo}

O público alvo do projeto Aprenda a Programar Jogando contempla crianças e jovens de diferentes idades, compreendendo desde o ensino fundamental ao ensino médio, em escolas públicas e privadas. O projeto, no entanto, não é restrito a esse público, e quando oportuno, é perfeitamente aplicável a participação de pessoas de outras faixas etárias.

\section{Ferramentas Utilizadas}

Há uma ampla variedade de ferramentas disponíveis para o ensino da programação e fizemos uma seleção que priorizou os seguintes critérios: conceitos de programação básicos abordados (comandos de condição e repetição), disponibilidade na língua portuguesa e acesso gratuito. As ferramentas escolhidas foram:

- Hour of Code ${ }^{3}$ : essa ferramenta possui o diferencial de estimular os alunos por meio de personagens conhecidos, por exemplo Minecraft, Frozen e Star Wars, e ao longo da atividade apresenta vídeos explicativos sobre os conceitos de programação como comandos de repetição e condicionais.

\footnotetext{
${ }^{3}$ https://code.org/learn
} 
- Scratch ${ }^{4}$ : plataforma onde os usuários podem criar suas histórias, jogos e animações, unindo blocos de comando de forma lógica. Estimula os alunos a explorarem sua criatividade e usa comandos de: condição, repetição, movimento, som, desenho, variáveis, sensores, operadores lógicos, entre outros.

- CodeMonkey ${ }^{5}$ : jogo educativo onde os alunos aprendem a criar códigos em uma linguagem de programação real, chamada CoffeeScript, sem necessitar conhecimentos prévios. O tutorial usado pelo projeto é a versão gratuita que aborda: condicionais, repetição, funções e variáveis.

- Monster Coding ${ }^{6}$ : neste jogo o usuário pode criar seu próprio personagem e guialo usando blocos de comando. São explorados nesta plataforma: booleanos, matrizes, condicionais e repetição.

- MIT App Inventor ${ }^{7}$ : plataforma de introdução a programação e criação de aplicativos para Android por meio de blocos de comando. Após montar o layout e a programação do aplicativo, o aluno pode testar sua criação em um emulador de computador ou no próprio dispositivo Android. Alguns dos conceitos abordados são: condicionais, funções, operadores lógicos, repetição, variáveis.

\section{Estrutura das atividades}

A estrutura de atividade que é seguida tanto nos minicursos, que contemplam vários encontros, como em oficinas, que possuem aproximadamente duas horas de duração.

Cada aula dos minicursos ou oficinas tem caráter teórico-prático, que se inicia com uma introdução teórica dos conceitos de programação, e segue com a explicação sobre a atividade a ser realizada. Dessa forma os conceitos de programação são conhecidos previamente e exemplificados ao longo da experiência com a atividade. A estrutura das atividades é dividida em quatro etapas conforme ilustrado na Figura 1.

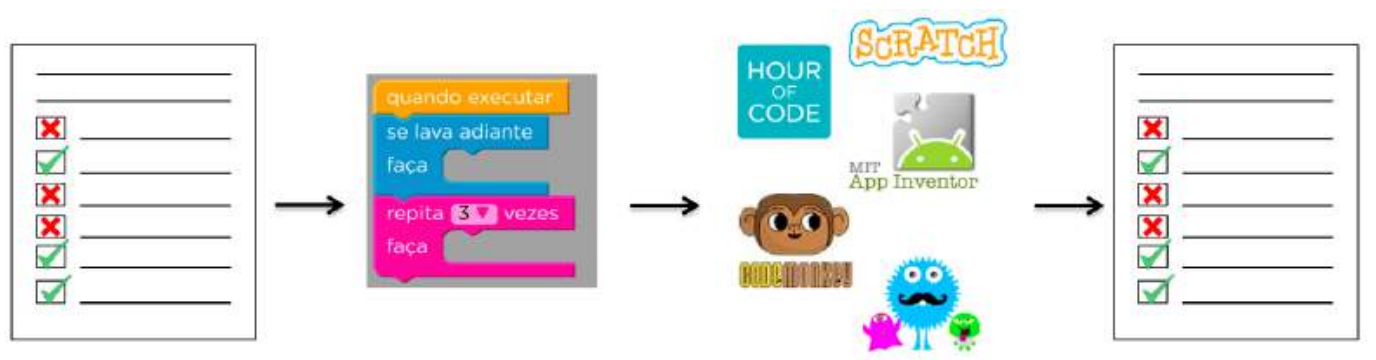

Conhecendo os alunos Computação desplugada Utilizando ferramentas Avaliando a atividade

Figure 1. Estrutura das atividades.

As atividades começam com uma conversa inicial para conhecer as expectativas da turma e logo em seguida os participantes respondem um questionário com perguntas sobre idade, alguns conceitos de computação e programação e o que esperam da atividade. Chamamos essa etapa de Conhecendo os alunos.

\footnotetext{
${ }^{4}$ https://scratch.mit.edu/

${ }^{5}$ https://www.playcodemonkey.com/

${ }^{6} \mathrm{http}: / /$ monstercoding.com/

${ }^{7}$ http://appinventor.mit.edu/explore/
} 
A etapa Computação desplugada tem como objetivo introduzir conceitos aos alunos. No início dos minicursos o conceito de algoritmo é apresentado e é realizada uma dinâmica em que eles são levados a controlar os movimentos de uma pessoa vendada, usando uma sequência de instruções verbais simples. Os outros conceitos (variáveis, comandos de condição e repetição, dentre outros) são apresentados quando utilizados pelas ferramentas.

A próxima etapa é a Utilizando as Ferramentas e as plataformas selecionadas são exploradas da seguinte forma:

- Criando Animações: a animação consiste em criar os movimentos de uma personagem. Utilizando o Scratch, os alunos possuem diversas possibilidade de personagens, planos de fundo e movimentos que podem ser utilizados.

- Jogando e aprendendo: nas ferramentas Hour of Code, Monster Coding e CodeMonkey os alunos são instruídos a concluir os tutoriais que proporcionam o entendimento da funcionalidade dos conceitos na solução de uma tarefa.

- Criando Jogos: o professor mostra um exemplo aos alunos e ensina sobre os comandos e conceitos. Os alunos são estimulados a desenvolverem seus próprios jogos personalizando e explorando novos comandos, personagens e figuras.

- Criando aplicativos: A turma é instruída como desenvolver o layout e o código do aplicativo na plataforma MIT App Inventor. A plataforma dispõe de inúmeros tutorias, onde essa equipe se inspirou ao desenvolver os tutoriais usados em aula.

A última etapa Avaliando das atividades é realizada após o uso das ferramentas e é aplicado um questionário perguntando sobre o nível de dificuldade e satisfação de forma geral. Sendo essa, uma oportunidade de os participantes expressarem suas opiniões e sugestões para o projeto.

\section{Relatos de Experiência e Avaliação das Atividades}

Nesta seção serão apresentados as oficinas e minicursos realizados e os resultados dos questionários respondidos. As atividades foram planejadas de forma que pudessem ser trabalhadas em oficinas e minicursos. As oficinas são realizadas em eventos de divulgação científica organizados pela UFRJ-Macaé e pela Fundação Educacional de Macaé (Funemac). A duração das oficinas é de aproximadamente duas horas e envolve um público diversificado. Já os minicursos são oferecidos na equipe do projeto na UFRJ-Macaé e em escolas parceiras. A duração dos minicursos varia de sete a dezesseis horas distribuídos ao longo de vários encontros.

\subsection{Oficinas}

Na tabela 1, temos a relação das oficinas, ferramentas utilizadas, faixa etária dos participantes de cada oficina e quantidade de participantes. Foram realizadas um total de quatro oficinas todas com ajuda de divulgação da UFRJ-Macaé e conseguimos atingir uma ampla faixa etária, desde os 10 aos 44 anos de idade, e um total de 71 participantes.

Nas oficinas realizadas na Semana Nacional de Ciência e Tecnologia (SNCT) ${ }^{8}$ e na Feira do Conhecimento ${ }^{9}$ foram utilizados os tutorias Pegue o Android ${ }^{10}$ e Calcule seu

\footnotetext{
${ }^{8}$ SNCT realizada na UFRJ-Macaé

${ }^{9}$ Feira do Conecimento foi organizada pela Funemac na UFRJ-Macaé

${ }^{10} \mathrm{https} / / /$ sites.google.com/site/projetoaprendaprogramar/tutoriaislinks/pegueandroid
} 
Table 1. Oficinas realizadas.

\begin{tabular}{|c|c|c|c|c|}
\hline Evento & Oficina & Ferramenta & Faixa etária & \# alunos \\
\hline $\begin{array}{l}\text { Inverno com } \\
\text { Ciência }\end{array}$ & $\begin{array}{l}\text { Aprenda a Progamar } \\
\text { Jogando }\end{array}$ & Hour of Code & Todas & 25 \\
\hline SNCT & $\begin{array}{l}\text { Aprenda a Progra- } \\
\text { mar seu Primeiro } \\
\text { App Android }\end{array}$ & MIT App Inventor & Jovens & 24 \\
\hline $\begin{array}{l}\text { Feira do Co- } \\
\text { nhecimento }\end{array}$ & $\begin{array}{l}\text { Aprenda a Progra- } \\
\text { mar seu Primeiro } \\
\text { App Android }\end{array}$ & MIT App Inventor & Jovens & 12 \\
\hline SECCOMP & Hour of Code & Hour of Code & Todas & 10 \\
\hline
\end{tabular}

$I M C^{11}$ realizados na ferramenta MIT App Inventor. Esses tutoriais foram desenvolvidos pela equipe do projeto considerando a duração e o público do evento e estão disponíveis online no website do projeto. Já nas oficinas realizadas durante o Inverno com Ciência ${ }^{12}$ e Semana de Educação em Ciência da Computação (SECCOMP) ${ }^{13}$ foi utilizada a ferramenta Hour of Code e os participantes podiam escolher qual tutorial disponível mais os interessavam. O tutorial Minecraft foi escolhido por 53,8\% dos participantes.

Foi a primeira vez que esses 71 participantes tiveram contato com a programação de computadores e após utilizarem a ferramenta no dia da oficina $100 \%$ dos participantes afirmaram que a atividade auxilia na compreensão dos conceitos de programação e recomendariam a oficina para alguém. Além disso, 100\% dos participantes também disseram ter interesse em aprender mais sobre a programação. Os participantes classificaram a sua experiência na oficina em uma escala que variando entre excelente e ruim e o resultado está na Figura 2. Como podemos observar aproximadamente $90 \%$ dos participantes do Inverno com Ciência e da SNCT classificaram como excelente e 90\% dos participantes da SECCOMP e da Feira do Conhecimento classificaram como excelente ou ótima. Nenhum participante classifcou como sendo uma experiência ruim.

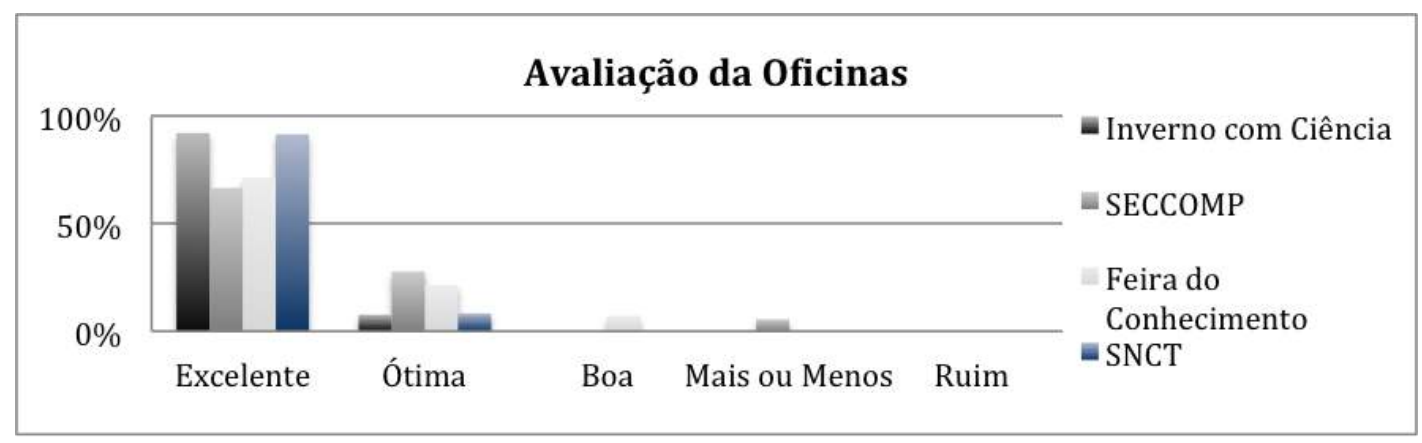

Figure 2. Avaliação das oficinas feitas pelos alunos participantes.

\footnotetext{
${ }^{11} \mathrm{https} / / /$ sites.google.com/site/projetoaprendaprogramar/tutoriaislinks/imc

${ }^{12}$ Inverno com Ciência foi organizado e realizado na UFRJ-Macaé.

${ }^{13}$ SECCOMP foi realizada na UFRJ-Macaé.
} 
Table 2. Oficinas realizadas.

\begin{tabular}{ccccc}
\hline Turmas & Escola & Faixa Etária & \# alunos & Carga Horária \\
\hline Turma I & Colégio A & Crianças & 167 & $7 \mathrm{~h}$ \\
Turma II & Colégio B & Jovens & 35 & $16 \mathrm{~h}$ \\
Turma III & Colégio B e C & Jovens & 25 & $8 \mathrm{~h}$ \\
\hline
\end{tabular}

\subsection{Minicursos}

Os minicursos foram oferecidos em três escolas de Macaé. Sendo que, no colégio A foi realizado com crianças do ensino fundamental e os colégios $\mathrm{B}$ e $\mathrm{C}$ as atividades foram desenvolvidas com alunos do ensino médio. Cerca de 227 alunos participaram dos minicursos oferecidas pelo projeto. Na tabela 2 temos a relação das turmas de minicursos e seus participantes.

Dividimos o planejamento considerando a faixa etária: classes infantis e classes juvenis. A principal diferença é como as ferramentas são exploradas em cada classe, onde a infantil possui ênfase na criação de jogos e animações usando programação em bloco. Por outro lado, a classe juvenil tem ênfase na criação de aplicativos para Android, tendo a introdução a programação criando animações e jogos.

Para os integrantes do colégio A a duração do curso foi definida em acordo com a disponibilidade da escola parceira que ofertou o laboratório de informática para os encontros quinzenais durante três meses. Foram realizadas um total de oito turmas contemplando 167 alunos. Para fins de explicação e avaliação chamaremos as oito turmas de Turma I, sem perda de generalidade. Nas classes infantis, a meta foi apresentar a área da programação, suas utilidades e conceitos básicos, de forma lúdica e divertida. A programação das atividades das aulas da Turma I foi:

- Scratch - Anime seu Nome: os alunos se familiarizam com a ferramenta utilizando comando básicos para criar uma simples animação com as letras de seus nomes.

- Monster Coding: realizando este tutorial auto guiado, os alunos aprendem sobre booleanos, condicionais e repetição.

- Hour of Code: os alunos escolhem entre os tutoriais: Frozen, Star Wars e Minecraft. Onde aprendem a funcionalidade dos comandos de repetição e condição.

- Scratch - Hide and Seek: inspirado no tutorial disponível no site do Scratch, os alunos usam comandos de repetição para criar um jogo de esconde-esconde com comandos de repetição, condicionais e outros.

- Code Monkey: já familiarizados com os blocos e princípios da programação, os alunos são apresentados a programação escrita através deste jogo educativo.

- Scratch - Jogo Pegue o Gato: esse jogo desenvolvido pela equipe, onde um gato guiado pelo mouse do computador, deve capturar todos os ratos. Este tutorial utiliza os conceitos: variáveis, condição, repetição e operadores lógicos.

Dos participantes dos minicursos oferecidos $82 \%$ não sabiam como um programa de computador era feito e nunca tinham ouvido falar em programação de computadores e ciência da computação. No final do minicurso ao responder a avaliação final 80,3\% dos alunos disseram ter interesse em aprender mais sobre ciência da computação. 
Os participantes avaliaram também o nível de dificuldade das ferramentas. $\mathrm{Na}$ Figura 3 podemos observar que as ferramentas Hour Of Code e Scratch foram classificados como nível médio pela maioria dos alunos. Já as ferramentas Monster Coding e CodeMonkey foram classificados como muito fácil pela maioria dos alunos. Uma pequena parcela dos alunos, menos de 5\% acharam as ferramentas com nível muito difícil.

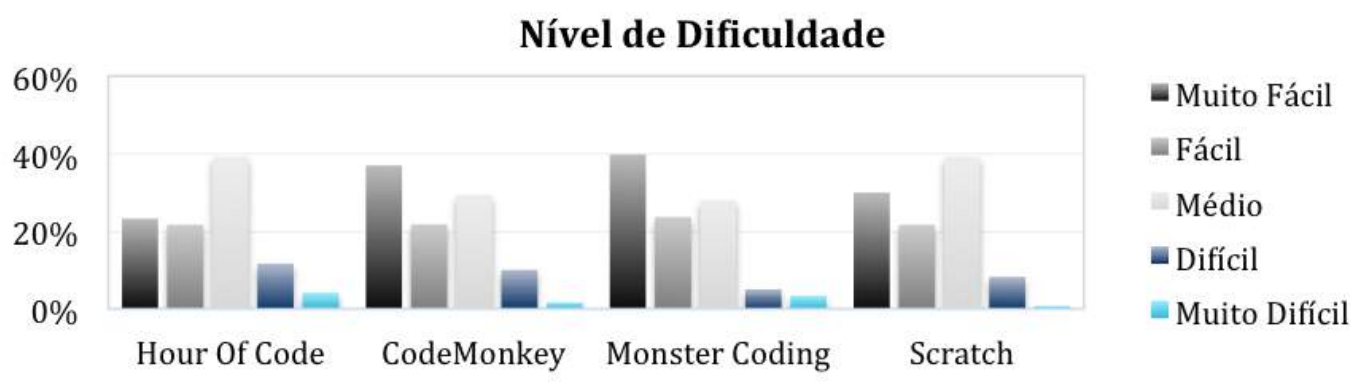

Figure 3. Avaliação do nível de dificuldade realizada pela Turma I

As ferramentas preferidas, Figura 4, foram CodeMonkey, Hour of Code e Scrach, nessa ordem. A que foi menos votada como favorita foi a Monster Coding. Interessante observar que Hour of Code e Scratch foram consideradas ferramentas de nível médio pelos alunos e mesmo assim foram favoritas. A ferramenta Code Monkey, que foi escolhida como favorita por mais de 50 alunos, utiliza nas tarefas a programação escrita e foi muito bem aceita pelos alunos que demostraram entendimento sobre a estrutura dos códigos.

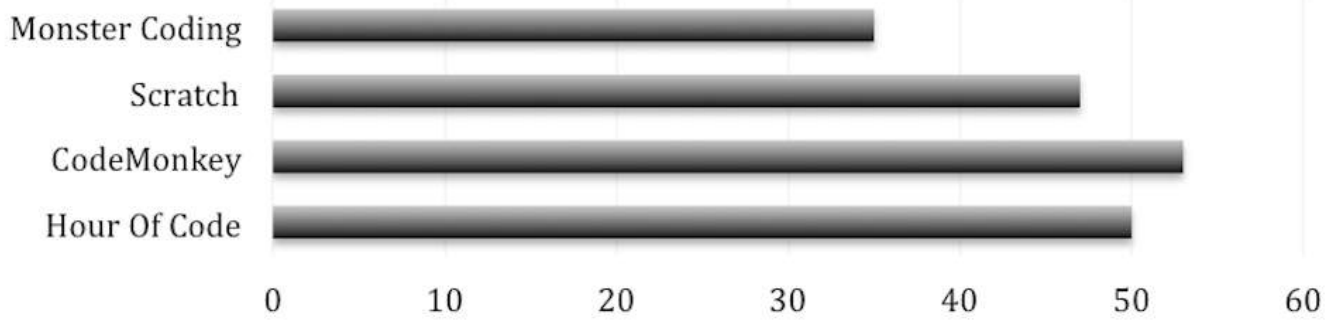

Figure 4. Ferramentas preferidas pela Turma I

Em relação as classes juvenis, os minicursos foram divulgados pelos participantes do projeto nas salas de aula dos colégios B e C. Porém, estas escolas não possuem laboratório de informática, então as aulas ocorreram nos laboratórios da UFRJ-Macaé e alunos de outras instituições integraram-se ao projeto. Nas Turmas II e III o nosso objetivo foi apresentar a programação de computadores ao utilizar as ferramentas e habilitar os participantes a criarem seu próprio aplicativo para Android. A programação das aulas da classe juvenil é:

- Scratch - Anime seu nome: a mesma aula introdutória dada nas classes infantis.

- Scratch - Jogo de Coletar: na criação desde jogo de coletar, os alunos utilizam conhecimentos de comandos de repetição, de condição e variáveis.

- Hour of Code: os alunos escolhem entre os tutorias disponíveis. 
- MIT App Inventor - Talk To $M e^{14}$ criação um aplicativo simples onde o celular reproduz o texto digitado pelo usuário. São usados nesta atividade: variáveis e recursos de caixa de texto e texto para falar.

- MIT App Inventor - Calcule seu IMC: usando comando condicionais e variáveis, os alunos criam um aplicativo onde o usuário pode calcular o seu IMC. São usados: condicionais, variáveis, operadores lógicos e recursos de caixa de texto.

- MIT App Inventor - Einstein Responde ${ }^{15}$ o usuário faz uma pergunta ao Albert Einstein, e ao sacudir o dispositivo, obtêm uma resposta. Ao desenvolver esse tutorial, o aluno entende o funcionamento de uma lista.

- MIT App Inventor - Pegue o Android: criação do jogo aonde aparece um bonequinho e o usuário ganha pontos quando consegue clicar. São usados conceitos de: variáveis, condicionais, repetição, operadores lógicos.

- MIT App Inventor - Crie seu aplicativo: os alunos devem criar seu próprio aplicativo. O aluno deve usar a criatividade para pensar em um tema, planejar as telas do aplicativo e pôr em pratica os conhecimentos obtidos no curso.

A última etapa, Crie seu aplicativo, só foi possível realizar com a Turma II pois na turma III a carga horaria foi reduzida e os alunos não puderam realizar essa atividade. Contudo, os alunos foram instruídos como desenvolver seu próprio aplicativo e os exemplos da Turma II foram mostrados.

Através dos questionários iniciais feitos nas turmas juvenis, foi constatado que mais de $50 \%$ dos alunos nunca tinha ouvido falar em ciência da computação e mais de $70 \%$ não sabia como um programa de computador é feito. Estes dados evidenciam a importância da implementação da programação nas escolas e as novas oportunidades que iniciativas como esta proporcionam. Além de mostrar para esses jovens uma possibilidade de carreira profissional.

Algumas atividades da ferramenta Scratch e Hour of Code foram realizadas tanto nas turmas infantis e juvenis e apesar da diferença de idade dos participantes não houve dificuldade dos mais novos em realizar as atividades. Pelo contrário, algumas crianças, mais entusiasmadas, realizaram a atividade com extrema eficiência.

A Figura 5 mostra um dos resultados do questionário, onde os alunos das Turmas I, II e III classificaram a sua experiência no minicurso. O resultado das Turmas II e III encontram-se juntos. Quase 100\% dos jovens classificaram o minicurso como excelente e mais de $50 \%$ das crianças.

\section{Considerações Finais}

Esse artigo apresentou as atividades realizadas pelo projeto Aprenda a Programar Jogando cujo objetivo é introduzir o conhecimento da programação de computadores a crianças e jovens. O projeto realizou oficinas e minicursos, e apresenta como contribuições a seleção de ferramentas disponíveis gratuitas online, a criação de uma estrutura de atividades que podem ser utilizadas tanto na realização das oficinas quanto na realização dos minicursos e um plano de aulas para ser utilizado em classes infantis e juvenis.

A seleção de ferramentas amigáveis, que utilizam temas e personagens conhecidos como Star Wars, Minecraft e Frozen para o desenvolvimento da didática proposta, foi

\footnotetext{
${ }^{14} \mathrm{https}$ //sites.google.com/site/projetoaprendaprogramar/tutoriaislinks/talk-to-me

${ }^{15} \mathrm{https}$ ://sites.google.com/site/projetoaprendaprogramar/tutoriaislinks/einstein-responde
} 


\section{Avaliação dos Minicursos}

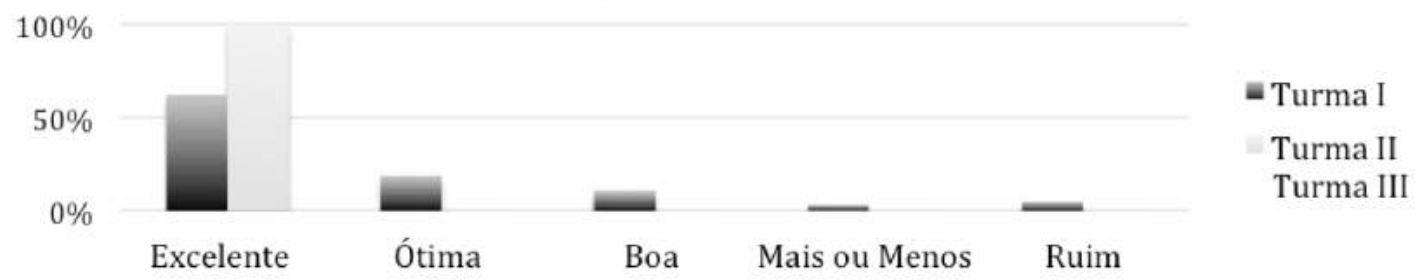

Figure 5. Nível de satisfação do minicurso pelas Turmas I, II e III

um fator relevante para o sucesso do projeto. Foi fundamental para incentivar os alunos a participarem das atividades, assim como para mantê-los motivados. Dessa foram, experiência resultou em um processo de aprendizado eficiente e prazeroso dos conceitos básicos de programação e algoritmos. Praticamente todos os 298 alunos que participaram das oficinas e atividades nunca haviam ouvido falar em ciência da computação e em programação de computadores e ao realizar a atividade, aproximadamente $90 \%$ dos participantes classificaram a experiência como sendo excelente ou ótima.

As ferramentas Scratch e Hour of Code foram utilizadas tanto nas turmas infantis e juvenis e, apesar da diferença de idade dos participantes, não houve dificuldade dos mais novos em realizar as atividades. Todos foram capazes de realizá-las, e inclusive, algumas crianças com pouca idade, mas com maior afinidade pelo assunto, realizaram a atividade com surpreendente eficiência. A proposta de criar aplicativos incentivou os jovens a participarem dos minicursos e diversos aplicativos foram desenvolvidos com foco em diferentes conceitos de programação. De modo geral, após o aluno ser introduzido à ferramenta o desenvolvimento é similar.

\section{Referências}

França, R. et al. (2014). A disseminação do pensamento computacional na educação básica. XXII Workshop sobre Educação em Computação (WEI)

Jakos, F., Verber, D. (2016). Learning Basic Programing Skills With Educational Games: A Case of Primary Schools in Slovenia. Journal of Educational Computing.

Oliveira, M. et al. (2014). Ensino de lógica de programação no ensino fundamental utilizando o Scratch. XXII Workshop sobre Educação em Computação (WEI).

Ramos, N. et al. (2015). Ensino de Programação para Alunas de Ensino Médio: Relato de uma Experiência. XXIII Workshop sobre Educação em Computação (WEI).

Resnick, M. et al. (2009). Scratch: programming for all. Communications of the ACM, v. 52, n. 11, p. 60-67.

SBC. (2015) Programa Meninas Digitais. Sociedade Brasileira de Computação. Disponível em: www.sbc.org.br. Acesso em: fevereiro/2017.

Silva, L., Carvalho, D. (2016). Saberes D’Avó: Uma Abordagem para o Ensino de Programação no Ensino Médio. XXII Workshop de Informática na Escola (WIE).

Wing, J. M. (2006). Computational thinking. Communications of ACM 49, 3, 33-35. 